\title{
LOS MODELOS DE GÉNERO EN EL ALUMNADO UNIVERSITARIO ESPAÑOL: UN ENFOQUE DESDE LA AUTOPERCEPCIÓN Y LAS RELACIONES DE GÉNERO.
}

\author{
Chiara Santoro \\ Universitá degli studi di Firenze (Italia), \\ chiara.santoro@hotmail.it \\ Ma Carmen Monreal Gímeno. \\ Universidad Pablo de Olavide, Sevilla. \\ mcmongim@upo.es \\ https://doi.org/10.17060/ijodaep.2017.n1.v2.941
}

Fecha de Recepción: 23 Marzo 2017

Fecha de Admisión: 1 Abril 2017

\section{RESUMEN}

En esta ponencia se presentan los resultados relativos a la resistencia de los modelos de género normativos de masculinidad y feminidad en la autopercepción y las relaciones de género de los/las estudiantes españoles/as, como uno de los objetivos específicos de la investigación "Los modelos de género entre el alumnado universitario desde una perspectiva intercultural". Esta investigación, que es parte del proyecto europeo IRSES Gendercit, tiene como objetivo general el estudio desde una perspectiva intercultural de los actuales modelos de género en las y los estudiantes universitarios/as de Italia, España y Argentina. La misma se desarrolla a partir de un enfoque que entiende el género desde una perspectiva relacional y utiliza a los modelos de género como medidores de la resistencia de una cultura patriarcal que dificulta el conseguimiento de la igualdad sustancial entre hombres y mujeres, que en el contexto español promueve la ley $3 / 2007$, de 22 de Marzo. A continuación, se presenta el cuestionario sobre modelos de género, utilizado en esta investigación, y los resultados relativos a la autopercepción y a la percepción de los demás en la muestra española, compuesta por 198 estudiantes de grado.

En las conclusiones, se evidencia como la resistencia diferencial de los modelos de género en hombres y mujeres en la autopercepción de si, puede estar relacionadas con las modalidades y las formas que adquieren las relaciones de género entre los sexos y las causas de sus principales diferencias por sexo.

El análisis de estos elementos nos permite así profundizar en la comprensión de las resistencias y de las dinámicas del sistema sexo-género en un contexto educativo, como el universitario, tan importante para la construcción de una sociedad igualitaria. 


\begin{abstract}
This paper presents the results concerning the resistance of normative gender models of masculinity and femininity in the self-perception and gender relations of Spanish students, as one of the specific objectives of the research "gender models among university students from an intercultural perspective". This research, which is part of the IRSES Gendercit European project, has the general objective of study from an intercultural perspective current gender models in Argentinian, Italian and Spanish university students. This research is carried out from an approach that understands gender from a relational perspective and uses gender models as indicators of the resistance of a patriarchal culture that hinders the achievement of substantial equality between men and women that the law 3/2007, of 22 March, promotes in Spain. Furthermore, the questionnaire on gender models, used in this research, and the results related to self-perception and gender relations in the Spanish sample, composed of 198 undergraduate students, are presented.

In the conclusions, we show how the differential resistance of gender models in men and women in self-perception, may be related to gender relations' modalities and forms and to the causes of their main differences.

The analysis of these elements allows us to deepen the understanding of the resistances and dynamics of the sex-gender system in an educational context, such as the university, so important for the construction of a more egalitarian society.

Key words: gender equality, university students, self identity, gender relations.

\section{1. "LOS MODELOS DE GÉNERO EN ÁMBITO UNIVERSITARIO DESDE UNA PERSPECTIVA INTER- CULTURAL": UNA INVESTIGACIÓN A PARTIR DEL GÉNERO COMO CONCEPTO RELACIONAL Y CON UN ENFOQUE EN LA INTERVENCIÓN.}

La investigación "Los modelos de género en ámbito universitario desde una perspectiva intercultural" pretende analizar la resistencia de los modelos normativos de género de feminidad y de masculinidad entre población universitaria de Argentina, Italia y España. En esta ponencia, nos vamos a centrar en los datos españoles relativos a uno de los aspectos que se analiza en la investigación: la autopercepción y la percepción de los demás. En este sentido, se pretende subrayar como una cultura de género binaria, que sustenta el sistema sexo-género, se sigue reproduciendo en y a partir de la relación con uno mismo y con el otro/la otra, que delimita las posibilidades de lo que podemos ser y al mismo tiempo nos permite reconocernos a través de la identificación de lo que no somos.

Por un lado, como afirma Garaizabal, el género es una de las variables fundamentales de la definición de la identidad y "utiliza un proceso de reflexión y observación simultaneas que tiene lugar en todos los niveles del funcionamiento mental y por medio del cual el ser humano se juzga a sí mismo en función de cómo cree que le juzgan los otros comparándoles con ellos y con respecto a una tipología significativa para estos" (Valcuende y Blanco, 2003).

Por el otro lado, este juego de reconocimiento de mi y del otro, termina definiéndonos y limitando nuestras posibilidades expresivas, tanto en hombres como mujeres, ya que, como explica Bourdieu (2000), "al carecer de otra existencia que la relacional, cada uno de los dos sexos es el producto del trabajo de construcción diacrítica, a un tiempo teórico y práctico, que es necesario para producirlo como cuerpo socialmente diferenciado del sexo opuesto (desde todos los puntos de vista culturalmente pertinentes), es decir, como habito viril, por consiguiente, no femenino, o femenino, por consiguiente, no masculino."

Desde esta postura teórica, que define a las estructuras de género desde su matriz relacional, esta investigación surge a partir de su conexión con la intervención para la igualdad de oportunidad entre hombres y mujeres, sobre todo en el contexto español. 
Las políticas de género en ámbito universitario, recogidas por la Ley 3/2007, de 22 de Marzo, para la igualdad efectiva de mujeres y hombres, obligan cada universidad a desarrollar un Plan de Igualdad, donde se evidencian las principales medidas para la consecución de la igualdad, a partir de un diagnóstico previo (art. 45, Ley 3/2007).

Por ello, esta investigación pretende desarrollar una herramienta que integre en el diagnostico de la situación de igualdad en el ámbito universitario, el análisis de las resistencias de los modelos de género normativos como elementos claves para la erradicación de la desigualdad de género y la consecución de la igualdad substancial. Por otro lado, la inclusión de esta investigación en el proyecto europeo IRSES Gendercit (género y ciudadanía), ha permitido integrar la perspectiva intercultural, que nos permite contrastar la resistencia de los modelos en contextos universitarios de diferentes países, Italia, España y Argentina, además de analizar las buenas practicas realizadas para conseguir la igualdad substancial.

\section{OBJETIVOS DE LA INVESTIGACIÓN.}

Como hemos visto, el objetivo general de la investigación es: analizar los modelos de género existentes desde una perspectiva intercultural en los/las estudiantes universitarios/as de Argentina, Italia y España.

Para alcanzar el objetivo general se han desarrollado diferentes objetivos específicos, que se reflejan en la misma estructura del cuestionario presentado a continuación, que van ahondando en los diferentes ámbitos donde se reproducen de forma transversal los modelos de género. Los objetivos específicos son los siguientes:

1. Autopercepción y percepción de los demás: analizar la autopercepción de estudiantes universitarios en los tres países considerados en el estudio (Argentina, Italia, España) y como perciben que los demás les perciben.

2. Ámbito educativo: Estudiar las relaciones de género en el ámbito educativo en los tres países considerados en este estudio (Argentina, España, Italia).

3. Uso del tiempo. Estudiar las relaciones de género en el uso del tiempo y en las actividades desarrolladas en función del sexo en los tres países considerados en el estudio (Argentina, Italia, España).

4. Conciliación ámbito laboral/familiar/personal: Analizar la conciliación entre el ámbito personal/ familiar/laboral en función de sexo en los tres países considerados en el estudio (Argentina, Italia, España).

5. Relaciones afectivos/sentimentales/sexuales: Analizar las relaciones afectivo-sentimentales-sexuales en función del sexo en los tres países considerados en el estudio (Argentina, Italia, España).

En esta ponencia, nos iremos enfocando en la presentación de los resultados relativos al primer objetivo específico, relativo a la autopercepción y percepción de los demás en el contexto español.

\section{EL CUESTIONARIO SOBRE MODELOS DE GÉNERO. ENFOQUE SOBRE LA AUTOPERCEPCIÓN Y LA METÁFORA DEL ESPEJO.}

Esta investigación se desarrolla con una metodología cuantitativa. La elección de esta metodología ha estado directamente relacionada con el enfoque en la intervención y en el desarrollo de nuevas herramientas para la ampliación del diagnostico de la situación de igualdad entre hombres y mujeres previo al diseño de las políticas de género en ámbito universitario. Por otra parte, es evidente que medir una variable tan compleja y transversal como los modelos de género a través de un cuestionario conlleva sus limitaciones y aproximaciones. 
Para dar voz a esta multiplicidad y complejidad, sin perder el valor de los modelos de género como posible variable de medición de la situación de igualdad substancial entre hombres y mujeres se han utilizado tres estrategias diferentes, que corresponden a características propias de los modelos de género.

Los modelos de género son transversales, y se manifiestan en los diferentes ámbitos de la vida de cada uno/ cada una de nosotros, y en las relaciones que establecemos en los diferentes contextos con los demás.

Por esto, el cuestionario se compone de 6 apartados que corresponden a las diferentes esferas donde se manifiesta la adhesión a modelos hegemónicos de referencia, y a los objetivos específicos planteados: autodefinición y percepción de los demás, ámbito educativo, uso del tiempo, conciliación ámbito laboral/familiar/personal, relaciones afectivos/sentimentales/sexuales. En esta ponencia, nos vamos a enfocar en los resultados relativos al primer apartado.

"Los modelos de género son mapas por los cuales transitamos" (Valcuende y Blanco, 2003).

Como hemos visto, el género es también una variable individual, que se articula como una característica identitaria propia, en constantemente relación con otros elementos y con las elecciones individuales de cada uno/cada una. Para posibilitar la expresión de estos matices, cada apartado del cuestionario se divide a su vez en dos sub-apartados, el primero con ítems a los que se tiene que contestar según las experiencias personales, mientras en el segundo se pide expresar opiniones y creencias. Esta estrategia permite evitar o por lo menos detectar respuestas que sigan el discurso de acuerdo con lo "políticamente correcto" sobre la igualdad de género.

Los modelos de género afectan tanto a hombres como a mujeres y se establecen sobre una base relacional. Por este motivo, el cuestionario indaga la resistencia de ambos modelos de masculinidad y de feminidad en chicos y chicas, ya que tanto la persistencia de una visión estereotipada hacia si mismo/a como hacia el otro/la otra conllevan la reproducción de roles, expectativas, oportunidades diferenciadas por sexo.

\section{MUESTRA DE LA INVESTIGACIÓN Y ENFOQUE EN LA MUESTRA ESPAÑOLA.}

Como hemos visto, la investigación ha sido integrada en el proyecto IRSES Gendercit y ha tenido un alcance internacional, involucrando a 600 estudiantes universitarios pertenecientes a tres universidades diferentes, en particular 200 estudiantes argentinos/as (Universidad de Cuyo, Mendoza), 200 italianos/as (Universitá deglistudi di Firenze) y 200 españoles/as (Universidad Pablo de Olavide, Sevilla).

La muestra es descriptiva, post facto, correlacional e intercultural. Se han incluido en la muestra estudiantes de diferentes carreras, dividiendo la muestra en ramas: por cada universidad, se han incluido 100 estudiantes de la rama de ciencias duras y 100 estudiantes de la rama de ciencias sociales/humanidades, para tener una muestra equilibrada por sexo y para dar cuenta de las diferencias presentes en el mismo contexto universitario.

Respecto a la muestra española, en la que nos vamos a centrar en el análisis de los resultados, consta de un total de 198 estudiantes: 91 hombres y 107 mujeres, que han contestado presencialmente al cuestionario. El 98,1\% de la misma tiene menos de 25 años, y es estudiante de grado universitario, que se reúnen en dos ramas: Humanidades/sociales (estudiantes de Trabajo Social) y Ciencias duras (estudiantes de derecho y ciencias de las actividades físicas y deportes).

Como podemos destacar en la siguiente grafica, la distribución de hombres y mujeres de la muestra española evidencia como sigue existiendo una diferenciación por sexo en la elección de las ramas de estudio, de acuerdo con los roles normativos de masculinidad y feminidad. 
Grafica 1. Porcentajes desagregados por sexo de estudiantes en las diferentes ramas de estudios. Muestra española.

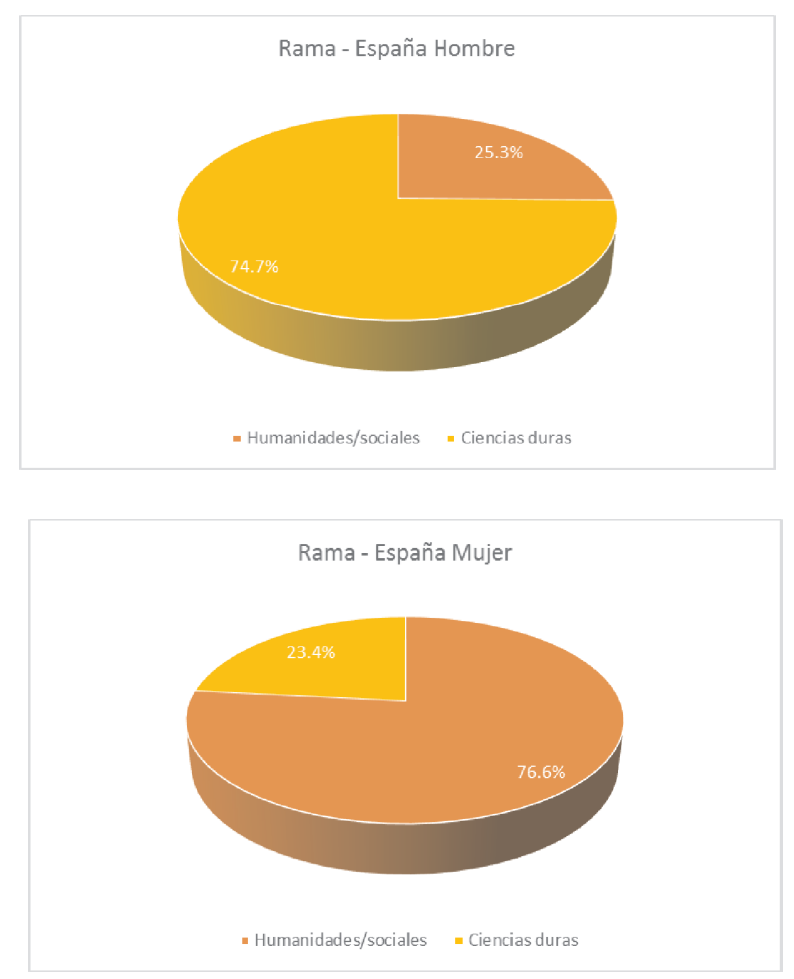

Las mujeres se concentran en la rama de humanidades y de sociales $(76,6 \%)$, mientras los hombres en la de las ciencias duras (74,7\%). Solo un $23,4 \%$ de mujeres elige estudiar carreras relacionadas con las ciencias dura, casi el mismo porcentaje $(25,3 \%)$ de los hombres que eligen dedicarse a las humanidades y a las ciencias sociales, porcentajes completamente invertidas.

Se destaca una total conformidad entre el sexo biológico afirmado por la muestra y su identidad de género, mientras respecto a la orientación sexual se evidencia una mayor variedades de elección, aunque con una fuerte prevalencia de la heterosexualidad, como se puede observar en la grafica siguiente. 
Grafica 2. Porcentaje desagregados por sexo relativos a la orientación sexual. Muestra española.
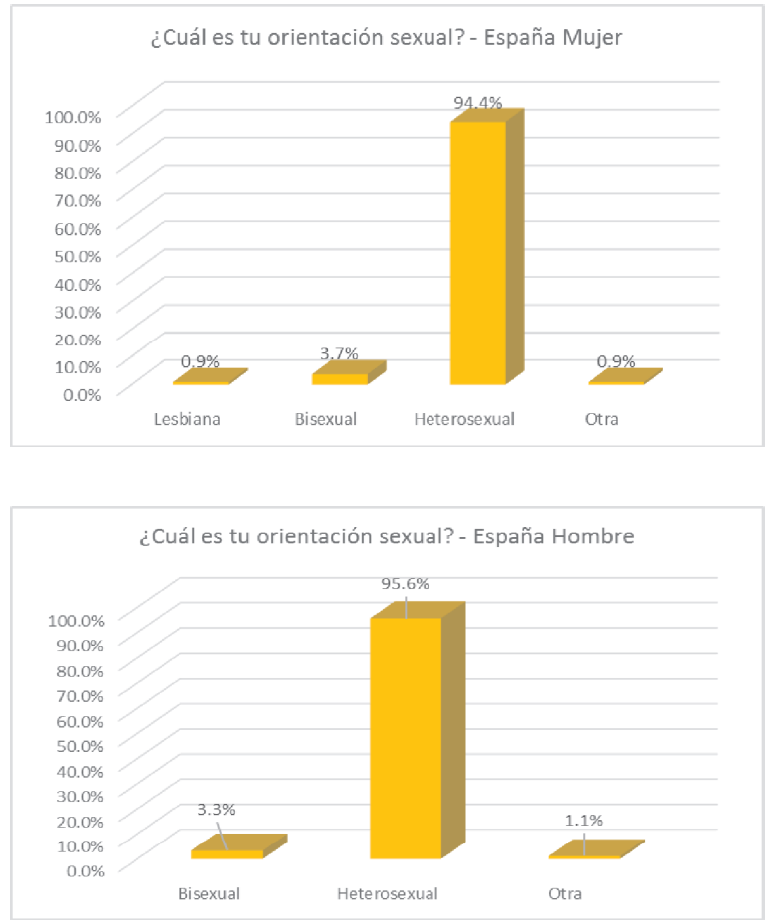

La mayoría de las mujeres $(94,4 \%)$ y de los hombres $(95,6 \%)$ de la muestra se definen heterosexuales. Se destaca un 3,3\% de bisexuales entre los varones y un $3,7 \%$ entre las mujeres. Entre las mujeres, un $0.9 \%$ se define lesbiana y un $0.9 \%$ afirma de tener otra orientación sexual. Entre los hombres, un 1,1,\% tiene otra orientación sexual respecto a las presentadas. Estos datos pueden claramente ser resultado de una cierta dificultad en expresar elementos relacionados con la identidad personal, sobre todo en un contexto en el que existe socialmente un general rechazo y estigma social respecto otras identidades de género y a una orientación sexual no conforme con el modelo heterosexual y cis- género.

Respecto a la autopercepción de la clase social de pertenencia, la mayoría de la muestra afirma pertenecer a la clase media, aunque con algunas diferencias por sexo, como podemos destacar en la grafica 3. 
Grafica 3. Porcentaje desagregados por sexo relativos a la autopercepción de la clase social de pertenencia. Muestra española.
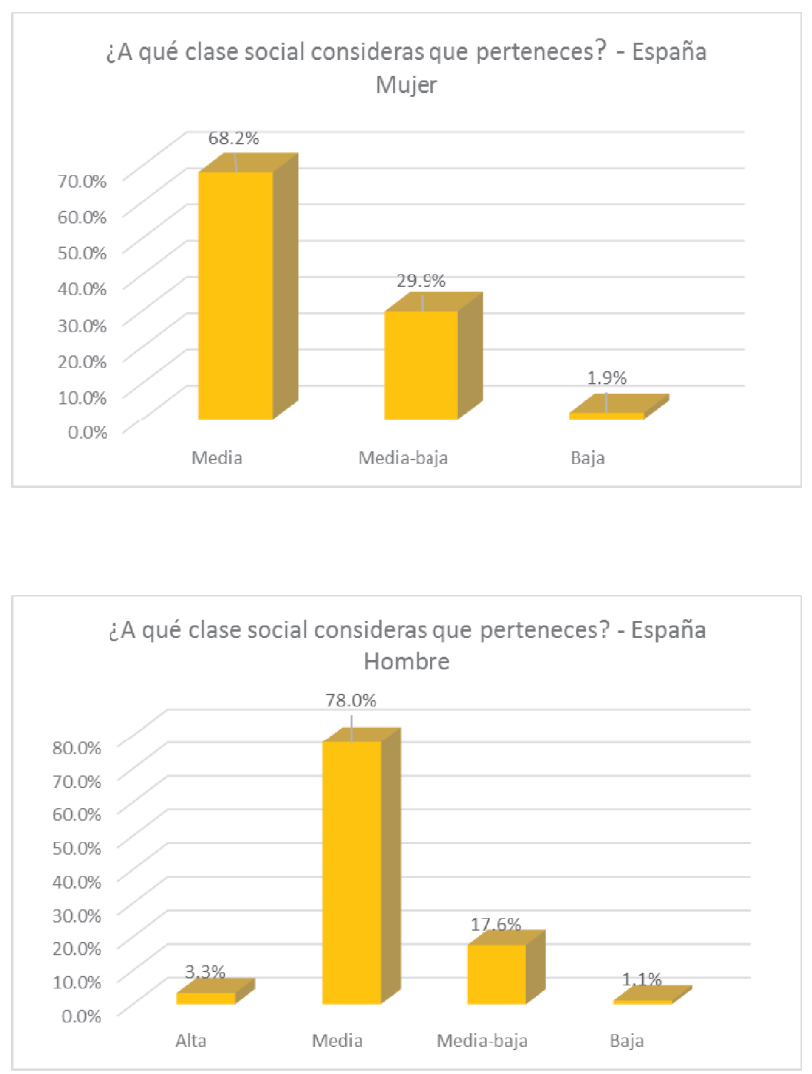

Como podemos observar, un $68,2 \%$ de mujeres se consideran pertenecientes a la clase media frente a un $78 \%$ de hombres. Ninguna mujer se siente perteneciente a la clase alta, mientras que un $30 \%$ se considera de la clase media-baja y un $1,9 \%$ de la clase baja. Por el contrario, un 3,3\% de los hombres se consideran perteneciente a la clase alta, un $17,6 \%$ a la clase media-baja y un $1,1 \%$ a la clase baja. Se destaca como, en general, las mujeres perciban pertenecer a clases sociales mas bajas respeto a los varones, y esto puede derivar del acceso real a bienes materiales y económicos de ellas o tambien a un escaso reconocimiento de las posibilidades de afirmacion social conectadas con sus profesiones y las de sus familias. Este dato puede relacionarse con lo relativo a las ramas de estudio (ver grafica 1) ya que la mayor concentracion de hombres en las ciencias duras podria resultar de una tradicion profesional familiar en estos ambitos, que conllevaria una mayor disponibilidad economica y una mayor percepcion de su relevancia social. 


\section{AUTO-REPRESENTACIÓN Y RELACIONES DE GÉNERO TE VEN TUS AMIGOS? IDAD CON HOMR. CAR EN LOS RESULTADOS RELATIVOS AL PRIMER APARTADO. NERO EL CONTE LA INVESTIGA- CIOIDAD CON HOMRENTRE ALUMNADO UNIVERSITARIO EN ESPAÑA.}

En esta ponencia, vamos a presentar los resultados relativos al apartado de autopercepcion y percepcion de los demas del cuestionario sobre modelos de género, anteriormente presentado, enfocandonos exclusivamente sobre la muestra española.

Para analizar esta realidad, partiendo del supuesto de que representa también y primariamente una variable identitaria personal, se ha propuesto a los/las entrevistados/as que elijan cinco entre 26 adjetivos para autodefinirse (item 1), que reflejan características normalmente asociadas a la masculinidad y a la feminidad. En segundo lugar, se le ha preguntado cómo creen que los/las definirían sus amigos (item 2) y como creen que los/las definirían sus amigas (item 3), con la posibilidad de elegir entre los mismos 26 adjetivos. $\mathrm{E}$

El primer item, está enfocado en analizar la resistencia de los modelos de género en la autopercepción, mientras que el item 2 y 3 nos permiten ahondar en como los modelos de género pueden afectar a las relaciones entre pares. Ademas, a través de estos tres ítems es posible comparar la autopercepción con su ajuste social según las relaciones de género que se establecen en el grupo de pares.

\subsection{Autopercepción y modelos de género.}

En la siguiente grafica se muestran los datos desagregados por sexo relativos a la autopercepción de la muestra española. Los porcentajes se refieren al primer adjetivo elegido por la muestra para definirse.

Grafica 4. "Me defino como una persona...". Porcentajes por primera elección. Muestra española.

\begin{tabular}{|l|l|l|}
\hline \multirow{2}{*}{ Categorías } & \multicolumn{2}{l}{ España } \\
\cline { 2 - 3 } & $M$ & $H$ \\
\hline Independiente & 7,8 & 13,1 \\
\hline Dependiente & 2 & 2,4 \\
\hline Dinámica/ Activa & 9,8 & 21,4 \\
\hline Tranquila & 3,9 & 8,3 \\
\hline Atenta con los demás & 11,8 & 3,6 \\
\hline Protectora & 2,9 & 1,2 \\
\hline Trabajadora & 16,7 & 11,9 \\
\hline Racional & 1 & 9,5 \\
\hline Cuidadora & -- & -- \\
\hline Posesiva & -- & -- \\
\hline Sensible & 9,8 & 3,6 \\
\hline Prudente & 6,9 & 3,6 \\
\hline Imprudente & -- & -- \\
\hline Maternal/ Paternal & 1 & -- \\
\hline Superficial & -- & -- \\
\hline Decidida & 1 & 1,2 \\
\hline Autoritaria & -- & -- \\
\hline Valiente & 2 & 1,2 \\
\hline Segura de sí misma & 2 & 3,6 \\
\hline
\end{tabular}




\begin{tabular}{|l|l|l|}
\hline Insegura & 5,9 & 1,2 \\
\hline Cariñosa & 2,9 & 7,1 \\
\hline Tozuda / Terca & 2 & -- \\
\hline Flexible & 1 & -- \\
\hline Agresiva & -- & -- \\
\hline Pacífica & 2,9 & 3,6 \\
\hline Comprometida & 6,9 & 2,4 \\
\hline Otras... & -- & 1,2 \\
\hline
\end{tabular}

Las estudiantes mujeres han elegido como primera opción: trabajadora $(16,7 \%)$, atenta con los demás $(11,8 \%)$, dinámica/activa y sensible $(9,8 \%)$ mientras que los estudiantes hombres han elegido prevalentemente: dinámica/activa $(21,4 \%)$, independiente $(13 \%)$ trabajadora $(11,9 \%)$, racional $(9,5 \%)$, evidenciando una marcada diferencia por sexo de pertenencia de los adjetivos seleccionada.

Además, a partir de los porcentajes, se subraya como los hombres tienen porcentajes de elección mas altos y elijan menos adjetivos para su definición (17 sobre 26) respecto a las mujeres (20 sobre 26), evidenciando como el modelo masculino resulte

ser mas homogéneo y menos variado.

En general podemos afirmar que priman adjetivos relacionados con el trabajo, dato que se puede explicar por la composición de la muestra, que reagrupa estudiantes universitarios/as que se están preparando para entrar en el mercado del trabajo.

\subsection{Relaciones con los demás y modelos de género.}

En la siguiente grafica se resumen los datos desagregados por sexo de la muestra española relativos a como creen que los definen amigos y amigas. Los porcentajes se refieren al primer adjetivo elegido por la muestra entre un listado de 26, ya proporcionado por el primer ítem.

Grafica 5. "¿Como me ven mis amigas? Como me ven mis amigos?" Porcentajes por primera elección. Muestra española.

\begin{tabular}{|c|c|c|c|c|c|}
\hline \multirow{2}{*}{\multicolumn{2}{|c|}{ Categorías }} & \multicolumn{2}{|c|}{$\begin{array}{c}\text { ¿Cómo te ven tus } \\
\text { amigas? }\end{array}$} & \multicolumn{2}{|c|}{$\begin{array}{c}\text { ¿Cómo te ven tus } \\
\text { amigos? }\end{array}$} \\
\hline & & $\mathbf{M}$ & $\mathbf{H}$ & M & $\mathbf{H}$ \\
\hline 1. & Independiente & 14,6 & 16,9 & 13,9 & 17,3 \\
\hline 2. & Dependiente & 1 & 3,9 & 1 & 3,7 \\
\hline 3. & Dinámica/Activa & 20,4 & 20,8 & 14,9 & 24,7 \\
\hline 4. & Tranquila & 10,7 & 10,4 & 6,9 & 12,3 \\
\hline 5. & Atenta con los demás & 8,7 & 6,5 & 11,9 & 6,2 \\
\hline 6. & Protectora & 1 & 2,6 & 4 & 1,2 \\
\hline 7. & Trabajadora & 10,7 & 7,8 & 10,9 & 8,6 \\
\hline 8. & Racional & 1 & 6,5 & 4 & 4,9 \\
\hline 9. & Cuidadora & -- & -- & -- & 1,2 \\
\hline 10. & Posesiva & 1 & -- & -- & 1,2 \\
\hline 11. & Sensible & 6,8 & 1,3 & 5,9 & 1,2 \\
\hline 12. & Prudente & 2,9 & 1,3 & 4 & 2,5 \\
\hline & Imprudente & 1,9 & -- & 1 & -- \\
\hline & Maternal/ Paternal & -- & -- & -- & 1,2 \\
\hline & Superficial & -- & -- & -- & -- \\
\hline 16. & Decidida & -- & 5,2 & 1 & 3,7 \\
\hline
\end{tabular}




\begin{tabular}{|ll|c|c|c|c|}
\hline 17. & Autoritaria & -- & -- & 1 & -- \\
\hline 18. & Valiente & 2,9 & -- & 1 & -- \\
\hline 19. & Segura de sí misma & 1,9 & -- & 1 & 2,5 \\
\hline 20. & Insegura & 2,9 & 2,6 & 1 & -- \\
\hline 21. & Cariñosa & 4,9 & 3,9 & 3 & 1,2 \\
\hline 22. & Tozuda / Terca & 1 & 2,6 & 3 & 1,2 \\
\hline 23. & Flexible & -- & -- & -- & -- \\
\hline 24. & Agresiva & -- & -- & 2 & -- \\
\hline 25. & Pacífica & 1 & 3,9 & 5 & 3,7 \\
\hline 26. & Comprometida & 3,9 & 3,9 & 2 & 1,2 \\
\hline 27. & Otras: & 1 & -- & 1 & -- \\
\hline
\end{tabular}

Los adjetivos mayoritariamente elegidos por las estudiantes mujeres respecto a como las ven sus amigas son: dinámica/activa (20,4\%), independiente (14,6\%), tranquila, trabajadora $(10,7 \%)$, atenta con los demás $(8,7 \%)$ mientras respecto a como las ven sus amigos son: dinámica $(14,9 \%)$, independiente $(13,9 \%)$, atenta con los demás $(11,2 \%)$, trabajadora $(10,9 \%)$.

En la percepción de los demás no hay fuerte variación de las estudiantes mujeres respecto a las relaciones con hombres y mujeres, aunque se refuerza el carácter de la atención hacia los demás $(8,7 \%-11,9 \%)$ y disminuye lo de la dinamicidad/actividad $(20,4 \%-14,9 \%)$ en el caso de las relaciones con varones.

En general, respecto a autopercepción, podemos afirmar que en las relaciones de género las mujeres tienden a reforzar el carácter de la tranquilidad, antes ausente y desaparece lo de la sensibilidad, aunque más fuerte en las relaciones con los hombres (9,8\% respecto al $6,8 \%$ )

Los adjetivos mayoritariamente elegidos por los estudiantes hombres respecto a como los ven sus amigas son: dinámica/activa (f.16), independiente (f.13), tranquila (f.8), mientras respecto a como los ven sus amigos son: dinámica (f.20), independiente (f.14), tranquila (f.10). Podemos afirmar que en el caso de los hombres las relaciones de género no influya en modo muy evidente en la percepción de los demás y hay una cierta conformidad con la propia autodefinición.

\section{DISCUSIÓN Y CONCLUSIONES.}

A partir de los dos análisis llevados a cabo, relativos a la autopercepción y a las relaciones con los demás, se pretende determinar la resistencia de los modelos de género normativos, analizando sus influencias en la definición de si y en las relaciones de los/las jóvenes universitarios/as, y en consecuencia, como se siguen reproduciendo, permitiéndonos plantear algunas propuestas de intervención.

\section{Autopercepción}

Respecto a los adjetivos elegidos, es evidente como el modelo español esté enfocado en la centralidad del trabajo, que tanto en hombres como en mujeres tiene un carácter central. En general, podemos afirmar que el modelo femenino se define por esta centralidad del trabajo en coexistencia con caracteres relacionados con la feminidad normativa (la atención con los demás, la sensibilidad), mientras que la auto representación de los hombres de la muestra pone el énfasis las características de dinamicidad, actividad, racionalidad que responden al modelo de masculinidad normativa.

En general podemos afirmar que en la autopercepción de estudiantes universitarios/as, siguen estando muy anclados en los modelos de género tradicionales, sobre todo en el caso de los varones. En las mujeres podemos destacar un cambio en el modelo normativo de feminidad relacionado con la inserción de las mujeres en el mercado laboral y la influencia del movimiento feminista en 
el valor que se atribuye hoy en día a la independencia. Se asiste, de alguna forma, a un giro hacia aspectos de un modelo masculino, mas valorado socialmente.

\section{Relaciones con los demás.}

Del análisis, se puede concluir que las mujeres españolas parecen tener una percepción de cómo las ven sus amigas mucho mas coherente con su auto representación respecto a la relativa a sus amigos. En particular, la elección de los adjetivos subraya como, según ella, en la relación con los hombres se evidenciarían aspectos mas asociados con el modelo de feminidad tradicional.

Los hombres no parecen poner en discusión su forma de ser en las relaciones con los demás, siendo su autopercepción coherente con la definición que según ellos darían amigos y amigas

Este elemento evidencia como la presión social sigue siendo más fuerte en el contexto social 0 por lo menos más percibida por las mujeres. Analizados desde una mirada relacional, estos resultados son muy sugerentes en cuanto nos permiten evidenciar las resistencias de los modelos de género en población estudiantil de la educación superior, que ya ha pasado por los procesos de socialización tanto primarios como secundarios (familia, escuela) tanto a nivel individual como en sus relaciones con el grupo de pares.

Podemos evidenciar como en el contexto español si por un lado el modelo de feminidad normativo ha sido revisado gracias a los aportes del movimiento feminista y a las políticas de género para la igualdad, el modelo de masculinidad sigue sin variar en sus contenido y en su homogeneidad. Por el otro lado, el modelo de feminidad se ha desplazado y ha integrado valores culturalmente asociados a los modelos de masculinidad, debido a la fuerte influencia en España del feminismo de la igualdad y de una necesidad de entrar en posiciones de poderes, ancladas a una visión masculina, sin que la valoración social asociada a la masculinidad y a la feminidad fuera puesta en valor. Las mujeres siguen teniendo que adaptar su forma de expresarse a modelos de feminidad normativa a la hora de relacionarse con otros hombres, mientras que los hombres no parecen padecer esta presión social, actuando de forma mas conforme a si mismo, tanto con hombres como con mujeres. Este hecho parece explicar como en las relaciones de género se entrelazan relaciones de poderes y presiones sociales, que de alguna forma conllevan una homologación general hacia un modelo que corresponde con lo que se considera socialmente positivo y que parece estar estrechamente conectado con la necesidad de ganar espacios de poder por los que hay que luchar.

\section{REFERENCIAS BIBLIOGRÁFICAS.}

Barberá, E., Martínez, I. (coords.) (2005). Psicología y género. Madrid: Pearson education.

Beauvoir, S. (1981) El segundo sexo. Madrid: Aguilar Ediciones.

Bellucci, M. (1994) De los estudios de la mujer a los estudios de género: han recorrido un largo camino. En Fernández, A. (Eds.) La mujer en la imaginación colectiva, Barcelona: Paidos

Beltrán, E.,Maquieira,V. (coords) (2012). Feminismos: debates teóricos contemporáneos. Madrid: Alianza. III ed.

Biemmi, I. (2012), Educare alla parità, Roma: Conoscenza.

Bourdieu, P. (2000) La dominaciónmasculina. Barcelona: EditorialAnagrama.

CagnolatiA.,Pinto Minerva F., S. Ulivieri(Eds.) (2013). Le frontiere del corpo. Mutamenti e metamorfosi.Pisa: ETS

Campani G. (2016), Antropologia di genere, Torino: Rosenberg\&Sellier.

Carabí, Á., Segarra, M. (Eds). (2000). Nuevas masculinidades. Barcelona: Icaria.

García, A., Freire, M. (2003). Desarrollo del género en la feminidad y en la masculinidad. Madrid: Narcea. 
Heritier, F. (1996). Masculino/ Femenino. El pensamiento de la diferencia. Barcelona: Ariel.

Lomas, C. (2004). Los chicos también Iloran: identidades masculinas, igualdad entre los sexos y coeducación. Barcelona: Paidós.

Lorente, M. (2009). Los nuevos hombres nuevos. Los miedos de siempre en tiempo de igualdad. Barcelona: Destino.

Martin Casares, A. (2006). Antropología de género. Madrid: La cátedra ediciones.

Monreal, M.C. y Martínez, B. (2010). Esquemas de género y desigualdades sociales. En Amador, L. y Monreal, M.C. (Eds.), Intervención social y género.Madrid: Narcea. pp.73-94

Moya Morales, M. (1993) Categorías de género: consecuencias cognitivas sobre la identidad, Revista de Psicología Social, 8 (2), pp. 171-187.

Moya Morales, M., Páez, D., Glick, P., Fernández Sedano, I., Poeschl, G. (1997) Sexismo, Masculinidad-Feminidad y Factores Culturales. Revista electrónica de Emoción y Motivación, 8 (4), pp. $127-142$.

Palacios, M. (2012). El derecho a la igualdad, Salta (Argentina): EUNSA.

Piccone Stella S., Saraceno C., (compiladoras) (1996), La costruzione sociale del femminile e del maschile, Bologna: II Mulino.

Robledo, Á., Puyana, Y. (compiladoras). (2000). Ética: masculinidades y feminidades. Bogotá: Universidad Nacional de Colombia. Centro de EstudiosSociales.

Sánchez Palencia, C., Hidalgo, J. (editores) (2001). Masculino plural: construcciones de la masculinidad. Lleida: Universidad de Lleida.

Valcuende, J., Blanco, J. (Eds.) (2003). Hombres. La construcción cultural de las masculinidades. Madrid: Talasa

Varela, N. (2013) Feminismo para principiantes. Barcelona: B de Bolsillo. 